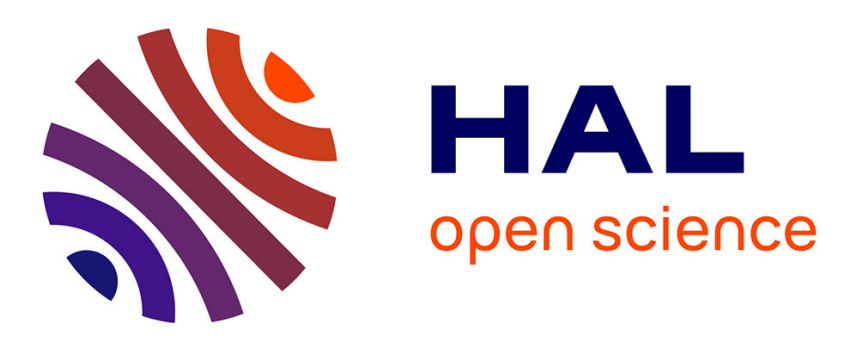

\title{
On the origin of cosmological magnetic fields by plasma instabilities
}

\author{
Reinhard Schlickeiser
}

\section{To cite this version:}

Reinhard Schlickeiser. On the origin of cosmological magnetic fields by plasma instabilities. 2005. hal-00002076v2

\section{HAL Id: hal-00002076 https://hal.science/hal-00002076v2}

Preprint submitted on 6 Jan 2005

HAL is a multi-disciplinary open access archive for the deposit and dissemination of scientific research documents, whether they are published or not. The documents may come from teaching and research institutions in France or abroad, or from public or private research centers.
L'archive ouverte pluridisciplinaire HAL, est destinée au dépôt et à la diffusion de documents scientifiques de niveau recherche, publiés ou non, émanant des établissements d'enseignement et de recherche français ou étrangers, des laboratoires publics ou privés. 


\title{
On the origin of cosmological magnetic fields by plasma instabilities - Revised Version
}

\author{
Reinhard Schlickeiser $\S$ \\ Institut für Theoretische Physik, Lehrstuhl IV: Weltraum- und Astrophysik, \\ Ruhr-Universität Bochum, Germany \\ E-mail: rsch@tp4.rub.de
}

\begin{abstract}
The existence of magnetic fields is a mandatory requirement for the onset of most nonthermal phenomena in cosmological sources especially gamma-ray burst sources and relativistic jet sources. The processes leading to the magnetization of the intergalactic medium are not yet known. Large-scale structures in the universe, like filaments and sheets of galaxies, evolve by the gravitational collapse of initially overdense regions giving rise to an intense relative motion of fully ionized gaseous matters and strong gaseous shock structures. We investigate analytically and numerically the generation of magnetic fields in the intergalactic medium by Weibel-type instabilities involving interpenetrating electron streams. Because of the hot temperatures of the intergalactic medium the investigation of the Weibel instability is based on the now available covariantly correct dispersion theory of linear waves, and thus improves on the existing non-relativistic treatments in the literature. These primordial Weibel magnetic fields may serve as cosmological seed fields for even stronger magnetic fields in cosmological sources.
\end{abstract}

\section{Introduction}

Today, magnetic fields are present throughout the universe and play an important role in many astrophysical situations. Our Galaxy, as many other spiral galaxies, is endowed with coherent magnetic fields ordered on scales $\geq 10 \mathrm{kpc}$ with typical strength $B_{G} \simeq 3 \cdot 10^{-6} \mathrm{G}$ [2, 1, 3, , 1 , or energy density relative to the cosmic microwave background radiation (CMBR) energy density $w_{\gamma}$

$$
\Omega_{G}=\left(B_{G}^{2} / 8 \pi\right) / w_{\gamma} \simeq\left(B_{G} / 3.2 \cdot 10^{-6} G\right)^{2} \simeq 1 \Omega_{\gamma}
$$

where $\Omega_{\gamma}$ is the density parameter in photons [5, 60. The galactic magnetic field is crucial for confining cosmic rays and transferring angular momentum away from protostellar clouds so that they can collapse and become stars. Magnetic fields also play an important role in the dynamics of pulsars, white dwarfs, and even black holes.

Elsewhere in the Universe, magnetic fields are known to exist and be dynamically important: in the intracluster gas of rich clusters of galaxies [7], in quasistellar objects and in active galactic nuclei. The existence of magnetic fields is a mandatory requirement for the onset of most nonthermal phenomena in cosmological sources especially gamma-ray burst sources and relativistic jet sources, as e.g. jet formation 
and collimation by MHD effects, acceleration of charged particles at magnetized shock fronts, synchrotron radiation.

More exotic, primeval magnetic fields are necessary to initiate substantial currents in superconducting cosmic strings [8], which if they exist may have important consequences for the production of ultrahigh-energy cosmic rays [9] and possibly for the initiation of structure formation [10].

The origin of cosmic magnetic fields is not yet known [11,3]. Many astrophysicists believe that galactic magnetic fields are generated and maintained by dynamo action [12] whereby the energy associated with the differential rotation of spiral galaxies is converted into magnetic field energy [13, 14, 15]. The dynamo mechanism is only a means of amplification and dynamos require seed magnetic fields. If a galactic dynamo has operated over the entire age of the galaxy $(\simeq 10 \mathrm{Gyr})$, it could have amplified a tiny seed field of $\simeq 10^{-19} \mathrm{G}$ although it is not yet clear 16 to what extent this process can work inpractice. Alternatively, initial fields of strength $B_{c} \simeq 10^{-9} \mathrm{G}$ can give rise to galactic fields of the observed values without a functioning dynamo mechanism: simple adiabatic compression of magnetic field lines during galaxy formation would amplify such initial fields to the present, observable values. Such fields have potentially interesting observational signatures on the CMBR, and if present would provide powerful constraints on models of the early universe. According to Eq. (1) a primeval magnetic field of $B_{c} \simeq 10^{-9} \mathrm{G}$ provides an energy density

$$
\Omega_{B}=10^{-7} \Omega_{\gamma}\left(B_{c} / 10^{-9} G\right)^{2}
$$

Since the universe through most of its history has been a good conductor [5] any primeval cosmic magnetic field will evolve conserving magnetic flux $B_{c} a^{2} \simeq$ const., where $a$ is the cosmic scale factor, implying that the dimensionless ratio $\Omega_{B}=$ $\left(B_{c}^{2} / 8 \pi\right) / w_{\gamma}$ for homogeneous (uniform or stochastic) magnetic fields remains approximately constant and provides a convenient invariant measure of magnetic field strength. Naively, from Eq. (2) one would expect a magnetic field of this amplitude to induce perturbations in the CMBR on the order of $10^{-7}$, which are about 1 percent of the observed CMBR anisotropies. The absence of such signatures may also serve as a consistency check on models of galaxy evolution that would be observationally incompatible with such large initial fields.

\section{Inhomogenous localized cosmological magnetic field from Weibel instabilities}

A localized process of cosmological magnetic field generation has recently been proposed 117, 18, 19 which occurs only in regions of intense gaseous streaming (and thus not homogeneously) at relatively late (redshift $z \leq 5$ ) stages of cosmological evolution. These localized intense gaseous streaming regions exhibit themselves in hydrodynamical simulations of a cold dark matter universe with a cosmological constant, which currently is considered to be the most successful theory for cosmological structure formation (e.g. [20,21]). Because large-scale structures in the universe, like filaments and sheets of galaxies, evolve by the gravitational collapse of initially overdense regions, they give rise to an intense relative motion of fully ionized gaseous matters (streaming speed of order $u \simeq 10^{4} \mathrm{~km} \mathrm{~s}^{-1}$ ) and baryonic density contrasts reaching $\alpha=n_{b} / n_{e} \leq 10$. Because the sound speed in a hot (with the temperature $T_{0}=10^{7} T_{7} \mathrm{~K}$ ) intergalactic medium is much smaller than the electron thermal speed, gaseous shock structures result. Schlickeiser and Shukla 
[19] demonstrated that besides the shock formation, the colliding streams of ionized matters efficiently generate long-lived quasi-static cosmological magnetic fields due to the Weibel instability [22] which operates in initially unmagnetized plasmas.

\subsection{The Weibel instability mechanism}

Fried [23] has given a simple exposition of the Weibel instability from counterstreaming electron distributions. Let us adopt the simple anisotropic distribution function

$$
f_{0}(\vec{v})=\delta\left(v_{x}^{2}-a^{2}\right) \delta\left(v_{y}\right) \delta\left(v_{z}\right)=\frac{1}{2} \delta\left(v_{y}\right) \delta\left(v_{z}\right)\left[\delta\left(v_{x}-a\right)+\delta\left(v_{x}+a\right)\right](3)
$$

to calculate the electron motion in the $z$-component of the infinitesimal magnetic fluctuation $\delta B_{z}=B_{1}(t) e^{\imath k y}$. From the equation of motion

$$
\frac{d v_{y}}{d t}=\frac{e}{m_{e} c} v_{x} \delta B_{z}
$$

we obtain for electrons with initially $v_{x}=a$ that

$$
\frac{d v_{y}}{d t}=\frac{e a}{m_{e} c} \delta B_{z}
$$

while electrons with initially $v_{x}=-a$ yield

$$
\frac{d v_{y}}{d t}=-\frac{e a}{m_{e} c} \delta B_{z}
$$

This gives rise to a change of thr flux of the $x$-component of momentum through a surface normal to $y$-axis of

$$
\begin{aligned}
& \frac{\partial}{\partial t}<v_{y} v_{x}>=\int d^{3} v f_{0} v_{x} \frac{d v_{y}}{d t}=\frac{e \delta B_{z}}{2 m_{e} c}\left[\int_{-\infty}^{\infty} d v_{x} v_{x} a \delta\left(v_{x}-a\right)\right. \\
& \left.-\int_{-\infty}^{\infty} d v_{x} v_{x} a \delta\left(v_{x}+a\right)\right]=\frac{e a^{2} \delta B_{z}}{m_{e} c}
\end{aligned}
$$

where $<>$ denotesd an average over the initial distribution (3). The gradient of this momentum flux causes a change in $\left\langle v_{x}\right\rangle$ given by

$$
\frac{\partial}{\partial t}<v_{x}>=-\frac{\partial<v_{y} v_{x}>}{\partial y}
$$

implying with Eq. (7)

$$
\frac{\partial^{2}}{\partial t^{2}}<v_{x}>=-\frac{\partial}{\partial y} \frac{\partial<v_{y} v_{x}>}{\partial t}=-\frac{e a^{2}}{m_{e} c} \frac{\partial \delta B_{z}}{\partial y}=-\frac{e a^{2}}{m_{e} c} \imath k \delta B_{z}
$$

Using Ampere's law for the current in $x$-direction

$$
j_{x}=-e n_{e}<v_{x}>=\frac{c}{4 \pi}(\operatorname{rot} \vec{B})_{x}=\frac{c}{4 \pi} \frac{\partial \delta B_{z}}{\partial y}=\frac{c}{4 \pi} \imath k \delta B_{z}
$$

yields

$$
<v_{x}>=-\frac{c}{4 \pi e n_{e}} \imath k \delta B_{z}
$$


Inserting Eq. (11) into Eq. (9) we then obtain

$$
\frac{\partial^{2} \delta B_{z}}{\partial t^{2}}=\frac{4 \pi e^{2} a^{2} n_{e}}{m_{e} c^{2}} \delta B_{z}=\frac{\omega_{p . e}^{2} a^{2}}{c^{2}} \delta B_{z}(t)
$$

with the growing solution

$$
\delta B_{z}(t) \propto \exp \left[\frac{\omega_{p . e} a t}{c}\right]
$$

For this reason the anisotropic distribution function (3) amplifies any fluctuating magnetic field component $B_{z}$. Obviously, such a magnetic field perturbation deflects the electron motion along the x-axis, resulting in current sheets $\left(j_{x}\right)$ which in turn amplify the perturbation. The amplified field lies in the plane perpendicular to the original electron motion.

\subsection{PIC simulation of this situation}

Lately, we [24] also simulated this instability with 2D3V-relativistic PIC simulations using a proton-electron mass ratio of $m_{p} / m_{e}=64$. As initial $(t=0)$ configuration we have chosen two counter-streaming electron-ion shifted Maxwellians with thermal velocities $v_{t h, e}=v_{t h, i}=0.1 c$ and with velocities $v_{d 1}=0.2 c$ and $v_{d 2}=-0.2 c$ in $x$-direction.

We investigated both a symmetric case with densities $n_{1}=n_{2}=50 /$ cell and an asymmetric case with $n_{2}=100 /$ cell $=2 n_{1}$. No initial electromagnetic fields were allowed. Our electron Debye length is $v_{t h, e} / \omega_{p e}=1.0 \Delta$, and the collisional skin depth $c / \omega_{p e}=10 \Delta$ (grid size $\Delta=1.0$ ) More details of the simulations will be given in Sect. 4 , but here we stress with Fig. 1 that indeed the $z$-component of the magnetic field rises with time in agreement with the qualitative arguments given above.

When the spatial scale of the excited fields is of the order of the electron gyroradius, the magnetic fields saturate due to the magnetic trapping of electrons in the wave potential. The saturated magnetic field turns out to be a fraction of microgauss at subequipartition level in cosmological environments.

\section{Analytical instability study}

The Weibel instability operates in initially unmagnetized plasmas. For a cold beam of density $n_{b}$ and bulk velocity $u$ propagating through hot electron-ion Maxwellian distribution of density $n_{e}$, the dispersion relation is ( [25], Eq. (6.1.5.15))

$$
\omega^{2}=-\omega_{b e}^{2}\left[\frac{k^{2} u^{2}}{k^{2} c^{2}+\omega_{b e}^{2}}-\frac{k^{2} v_{t h, e}^{2} v_{t h, p}^{2}}{\omega_{p e}^{2} v_{t h, p}^{2}+\omega_{p i}^{2} v_{t h, e}^{2}}\right]
$$

which leads to purely growing $\left(\omega^{2}=-\Gamma^{2}\right)$, i.e. aperiodic, transverse oscillations if

$$
\frac{u^{2}}{k^{2} c^{2}+\omega_{b e}^{2}}>\frac{v_{t h, e}^{2} v_{t h, p}^{2}}{\omega_{p e}^{2} v_{t h, p}^{2}+\omega_{p i}^{2} v_{t h, e}^{2}}
$$

For an equal temperature plasma $T_{e}=T_{p}=T_{0}$ this condition becomes

$$
\frac{u^{2}}{k^{2} c^{2}+\omega_{b e}^{2}}>\frac{v_{t h}^{2}}{2 \omega_{p e}^{2}}
$$




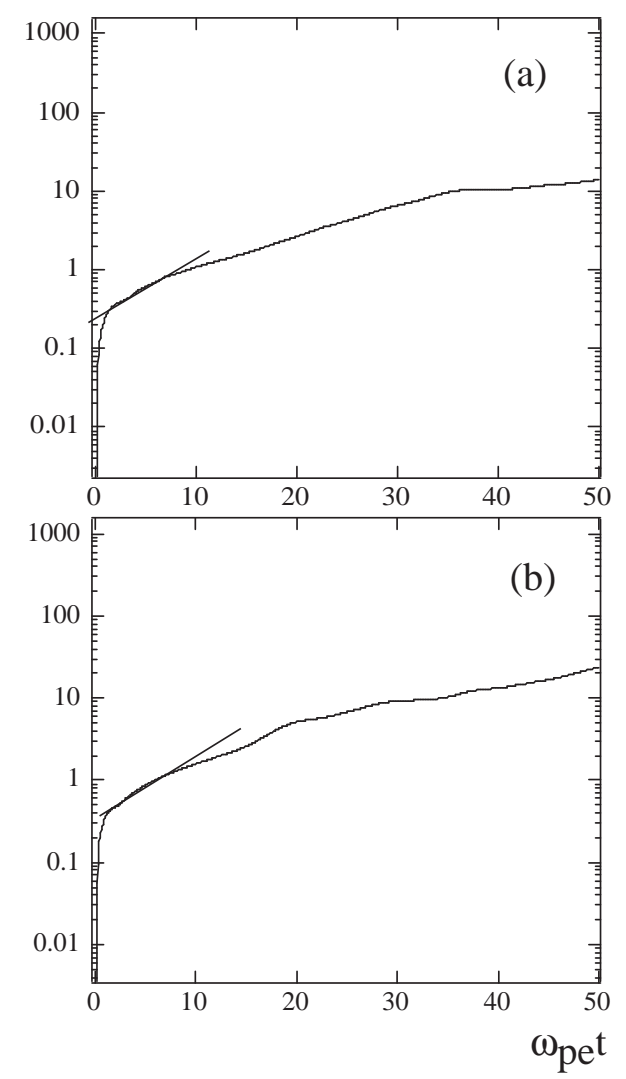

Figure 1. The time evolution of the magnetic field energy $\iint B_{z}^{2} d x d y$ during early stage:(a) symmetric case, and (b) asymmetric case. From 24$]$.

which is equivalent to the wavenumber restriction

$$
k^{2}<k_{0}^{2}=\frac{\omega_{p e}^{2}}{c^{2}}\left[\frac{2 u^{2}}{v_{t h}^{2}}-\frac{n_{b}}{n_{e}}\right]
$$

In order to have real wavenumbers it is required that

$$
u \geq u_{c}=\left(\frac{n_{b}}{2 n_{e}}\right)^{1 / 2} v_{t h}=8.73 \cdot 10^{3} \alpha^{1 / 2} T_{7}^{1 / 2} \mathrm{~km} \mathrm{~s}^{-1}
$$

i.e. for any given density contrast $\alpha=n_{b} / n_{e}$, this type of Weibel instability sets in, provided (1) that the streaming velocity $u$ is larger than $u_{c}$, and (2) that the beam is cold.

In the wavenumber regime $|k|<\left|k_{0}\right|$, the maximum growth rate is

$$
\Gamma_{\max }=\frac{\alpha v_{t h} \omega_{p e}}{2^{1 / 2} c}\left[\frac{u}{u_{c}}-1\right]^{3 / 4}
$$

However, this transverse Weibel instability will only be the primary relaxation mechanism for initial beam-plasma configuration, if its maximum growth rate is 
much larger than the growth rate of all other plasma instabilities in particular the longitudinal (Langmuir) two-stream instability with 26

$$
\Gamma_{\text {max }, \text { Langmuir }} \simeq \frac{3^{1 / 2}}{2^{4 / 3}} \alpha^{1 / 3} \omega_{p e}
$$

Comparing the last two equations we see that the maximum growth rate of the Weibel instability is smaller than maximum growth rate of Langmuir instability if

$$
\alpha\left[\frac{u}{u_{c}}-1\right]^{9 / 8}<115 T_{7}^{-3 / 2}
$$

i.e. the Langmuir instability is the primary relaxation mechanism, and the Weibel instability then sets in as a secular instability. If we identify the regions of large streaming velocities with strong non-radiative shocks with density contrast $\alpha=4$ and express the streaming velocity $u=M v_{t h} / 43$ in terms of the Mach number we obtain for Eq. (21)

$$
M<1192 T_{7}^{-4 / 3}
$$

Miniati ( 27]) has determined the distribution of shocks in baryonic flows resulting from cold dark matter simulations. The maximum Mach number values are below 200 indicating that high Mach number shocks violating condition (22) do not occur. Consequently, (i) the Weibel instability indeed occurs as a secular instability after full development the Langmuir instability, and (ii) the simultanous presence of electrostatic waves has to be expected.

\subsection{Secular Weibel instability}

The outcome of the electrostatic Langmuir instability is well known [28]: the beam distribution quickly relaxes to a plateaued distribution $\left(\frac{\partial f_{e}}{\partial p_{\|}}=0\right)$ in the initial direction of the beam. Non-resonant interactions then provide some energy exchange between the background plasma and the plateaued distribution in the $p_{\|}$-phase space direction.

Therefore we may characterize the plasma distribution after the Langmuir relaxation as anisotropic bi-Maxwellian

$$
f_{0}=\frac{n_{e}}{(2 \pi)^{3 / 2} u_{0}^{2} u_{3}} \exp \left[-\frac{v_{0}^{2}}{2 u_{0}^{2}}-\frac{v_{3}^{2}}{2 u_{3}^{2}}\right]
$$

where $u_{3}=v_{t h}$ and $u_{0}=u$ represents the plateaued distribution. This distribution corresponds exactly to case studied originally by Weibel [22]: in the limit $\omega /\left(u_{3} k\right)=$ $\omega /\left(v_{t h} k\right)>1$, corresponding to $u>v_{t h}$, the dispersion relation reads

$$
\omega^{4}-\left(\omega_{p, e}^{2}+k^{2} c^{2}\right) \omega^{2}-\omega_{p, e}^{2} u^{2} k^{2}=0
$$

and implies purely growing modes with the growth rate

$$
\Gamma_{4} \simeq \frac{u \omega_{p, e} k}{\left(\omega_{p, e}^{2}+k^{2} c^{2}\right)^{1 / 2}}
$$

The condition $\omega /\left(v_{t h} k\right)>1$ restricts the wavenumber to values smaller

$$
|k|<k_{s, \max }=u \omega_{p, e} /\left(v_{t h} c\right)
$$


so that the maximum growth rate is

$$
\Gamma_{4, \max }=\frac{u \omega_{p, e}}{c}=0.54 n_{-4}^{1 / 2} T_{7}^{1 / 2} M \quad \mathrm{~s}^{-1}
$$

The miminum growth time of the secular Weibel instability $\tau_{s}=\Gamma_{4, \text { max }}^{-1}$ then is much smaller than any cosmological time scale. In terms of the Mach number the instability condition $u>v_{t h}$ is equivalent to $M>43$.

\subsection{Estimate of saturated magnetic field value}

The free streaming of particles across the magnetic field lines is suppressed once the magnetic field amplitude has grown to a level that the particle's gyroradii in the excited magnetic fields, viz. $\rho=u / \Omega_{e}$, are comparable to the characteristic scale length of unstable modes, $k_{\mathrm{s}, \text { max }}^{-1}$, yielding

$$
B \simeq \frac{m_{e} u c k_{\mathrm{s}, \max }}{e}=\sqrt{4 \pi n_{e} m_{e}} \frac{u^{2}}{v_{t h}}
$$

This condition can be rewritten as

$$
\frac{B^{2} / 8 \pi}{n_{e} m_{e} u^{2}}=\frac{u^{2}}{2 v_{t h}^{2}}
$$

Computer simulations of the instability 29, 30,31,32, 24, confirm that saturation occurs at slightly subequipartition values of $B$,

$$
\frac{B_{s}^{2} / 8 \pi}{n_{e} m_{e} u^{2}}=\eta \frac{u^{2}}{2 v_{t h}^{2}}
$$

with $\eta \simeq 0.01-0.1$. Using $\eta=0.01$

$$
B_{s} \simeq 0.1 \sqrt{4 \pi n_{e} m_{e}} \frac{m_{e}}{m_{p}} v_{t h} M^{2}=1.3 \times 10^{-7} T_{7}^{1 / 2}\left(\frac{M}{43}\right)^{2} \mathrm{G}
$$

Consequently, the secular Weibel instability provides saturated magnetic field values over a rather wide range being determined by the distribution of Mach numbers of shock waves from cosmological structure formation with values larger than the instability condition $M>43$.

Taking $M=100$ as upper limit, the maximum field strength is $B_{s, \max } \simeq 7.0 \cdot 10^{-7}$ $\mathrm{G}$. This upper limit is consistent with the upper limit $\leq 10^{-6} \mathrm{G}$ in large-scale filaments and sheets, derived from rotation measure observations [33].

\subsection{Scales}

The analytical studies of the Weibel instability are formulated as an initial temporal instability analysis, assuming tacitly that the initial distribution functions (3) and (23) hold at all spatial scales up to $l_{\max }=\infty$. The Weibel analysis then yields aperiodic, i.e. purely growing, fluctuations at all wavenumbers $0 \leq k \leq k_{\max }$ where in the case of nonrelativistic thermal plasma temperatures [34, 35]

$$
k_{\max }=\frac{\omega_{p, e}}{c} \frac{\sqrt{1-\gamma^{2}}}{\gamma}
$$

where the anisotropy factor $\gamma=v_{\mathrm{th}, \|} / v_{\mathrm{th}, \perp}$ is limited to values $\left(v_{\mathrm{th}, \|} / c\right)<<\gamma<1$. 

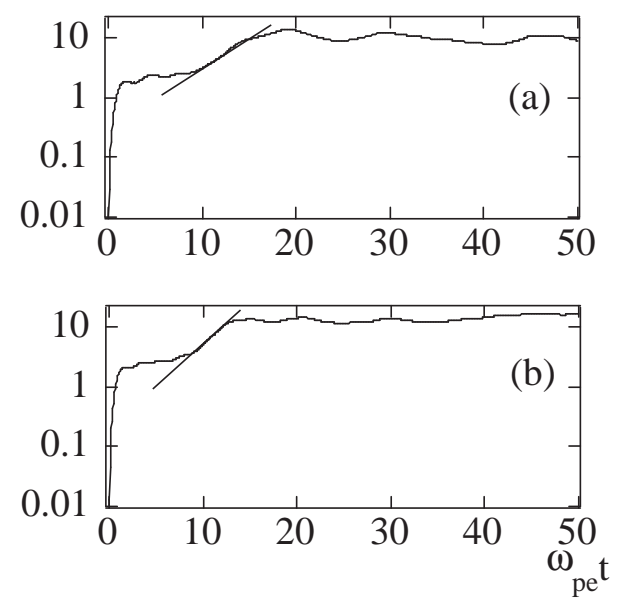

Figure 2. Time evolution of longitudinal electrostatic field energy $\iint E_{x}^{2} d x d y$ during early stage: (a) symmetric case, (b) asymmetric case. From 24$]$

In reality, however, the maximum spatial scale $l_{\max }$ will be finite, being set by the size of the localized intense gaseous streaming regions generated in the hydrodynamical simulations of the cold dark matter universe. These are of megaparsec order $l_{\max } \simeq 1$ Mpc $\left(1 \mathrm{Mpc}=10^{6} \mathrm{pc}=3.086 \cdot 10^{24} \mathrm{~cm}\right)[36,37$. We therefore expect spatial scales of the aperiodic Weibel fluctuations occurring over the large range of

$$
l_{\min } \leq l \leq l_{\max } \simeq 1 \mathrm{Mpc}
$$

with

$$
l_{\min }=\frac{2 \pi}{k_{\max }}=\frac{3.63 \cdot 10^{8}}{n_{-4}^{1 / 2}} \frac{\gamma}{\sqrt{1-\gamma^{2}}} \mathrm{~cm}
$$

where we adopt an intergalactic electron density of $n_{e}=10^{-4} n_{-4} \mathrm{~cm}^{-3}$. With the minimum value $\gamma=v_{\text {th }, \|} / c$ the smallest scale

$$
l_{\min }=2 \pi \lambda_{\mathrm{De}, \|}=1.37 \cdot 10^{7}\left(T_{7} / n_{-4}\right)^{1 / 2} \mathrm{~cm}
$$

is given by the parallel Debye length $\lambda_{\mathrm{De}, \|}=v_{\mathrm{th}, \|} / \omega_{p e}$. Obviously, the Weibel mechanism is capable to generate micro Gauss magnetic fields from the plasma Debye scale up to cosmological scales set by the large-scale structures formed in the cold dark matter universe.

\section{Simulation studies}

The particle-in-cell simulation results of Sakai et al. 24 quantitatively confirm the analytical results of Sect. 3, and provide corroborative evidence that the Weibel instability is a viable mechanism for the magnetization of the cosmological plasma.

We now provide more details of our computer simulation results. We used 2D3V, fully relativistic electromagnetic PIC code, modified from 3D3V TRISTAN code [38]. The system size was $L_{x}=16000 \Delta$ and $L_{y}=64 \Delta$, where $\Delta(=1.0)$ is the grid size. Periodic boundary conditions for both $\mathrm{x}$ - and $\mathrm{y}$-directions are imposed on particles and fields. The initial states of the plasma in the left-side region $(x \leq 8000 \Delta)$ and in 

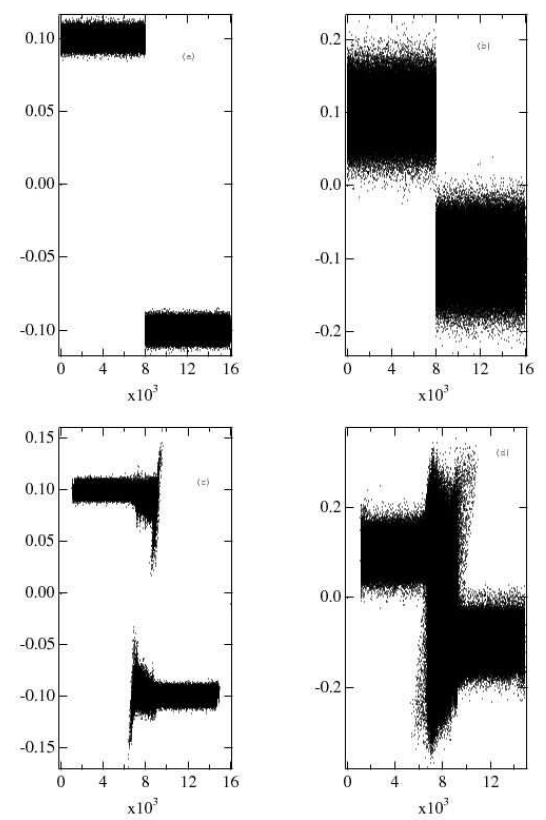

Figure 3. Phase-space plots of the protons $V_{i x} / c$, (a) $\omega_{p e} t=0$, (c) $\omega_{p e} t=600$, and the electrons $V_{e x} / c$, (a) $\omega_{p e} t=0$, (c) $\omega_{p e} t=600$. From [24].

the right-side region $(x>8000 \Delta)$ have the shifted Maxwellian with $v_{d}=+0.2 c$ and $v_{d}=-0.2 c$, respectively, where $c$ is the speed of light in vacuum. We performed two cases with equal density of $50 /$ cell for symmetric asymmetric cases where the rightside density $(100 /$ cell $)$ is twice larger than the left-side plasma density. The electron and ion temperatures are the same, so that the electron thermal speed is $0.1 c$. The chosen values especially of the streaming velocities $\pm 0.2 c$ should be regarded as typical values at rather early phases of the evolution of the universe.

Our simulation results are summarized as follows. Figure 1(a) displays the time evolution of the magnetic field energy $\iint B_{z}{ }^{2} d x d y$ during the early stage of symmetric density. Figure 1(b) exhibits the time evolution of the magnetic field energy $\iint B_{z}{ }^{2} d x d y$ during the early stage of asymmetric density. The solid lines in Figs. 2(a) and 2(b) show the linear growth rate. We can estimate the linear growth rate $\gamma / \omega_{p e}=0.1$ for the symmetric case, and $\gamma / \omega_{p e}=0.195$ for the asymmetric case.

Figure 2(a) shows the time evolution of the electrostatic electric field energy $\iint E_{x}{ }^{2} d x d y$ during the early stage of symmetric density. Figure 2(b) shows the time evolution of the electric field energy $\iint E_{x}^{2} d x d y$ during the early stage of asymmetric density. The solid lines show the linear growth rate. We can estimate the linear growth rate $\gamma / \omega_{p e}=1.03$ for symmetric case, and $\gamma / \omega_{p e}=1.49$ for the asymmetric case. This figure confirms the simultanous generation of electrostatic waves in the instability.

Figures 3(a) and 3(c) exhibit the phase-space plots of protons $\left(V_{i x}\right.$ is normalized by c) at $\omega_{p e} t=0$, and at $\omega_{p e} t=600$, respectively. Figures 3(b) and 3(d) depicts the phase-space plots of the electrons ( $V_{e x}$ is normalized by c) at $\omega_{p e} t=0$, and at $\omega_{p e} t=600$, respectively. Figure 4 displays the time history of the magnetic field energy $\iint B_{z}{ }^{2} d x d y$ normalized by the initial electron flow energy. 


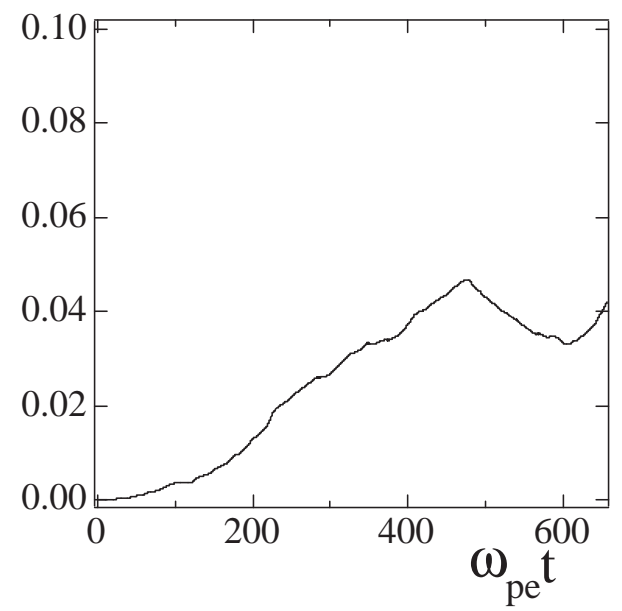

Figure 4. The time history of the magnetic field energy $\iint B_{z}^{2} d x d y$ normalized by the initial electron flow energy. From 24.

The estimates of the linear growth rates of the instabilities are in good agreement with the theoretical estimates of Sect. 3. Figure (6) shows energization of the electrons by Langmuir waves that are excited by counter-streaming electron clouds. The increased electron kinetic energy is associated with the electron heating. The conversion rate (the ratio between the magnetic field energy and the electron kinetic energy), as evident from Fig. (7), is about $5 \%$ for $v_{d}=0.2 c$. The magnetic field energy for $v_{d}=0.5 c$ is about ten times larger than that for the case $v_{d}=0.2 c$.

These PIC simulation prove the generation of magnetic fields in plasmas containing counter-streaming electron beams. The simulations used a proton-electron mass ratio of 64 . Since the mechanism involves both electrostatic and electromagnetic modes, it has to be checked in future work how this proton-electron mass ratio affects the saturation level of the transverse magnetic fields. With the present simulations we find that purely growing magnetic fields and oscillatory Langmuir oscillations develop in two different regimes. The growth rate of the purely growing magnetic fields in our simulations is in good agreement with the theoretical prediction. Linearly unstable magnetic fields saturate by trapping electrons in the vector potential. Counter propagating electron beam driven Langmuir waves are found to energize electrons. The ratio between the magnetic field energy and the electron kinetic energy is found to be five percent for $v_{0} / c=0.2$. This ensures that the generation of magnetic fields by colliding electron clouds is a fundamental process in cosmological plasmas.

\section{Covariant dispersion theory of the Weibel instability}

The good agreement of the linear growth rate of the Weibel instability obtained from the relativistic PIC simulations (Sect. 4) and from the analytical estimates of Sect. 3 deserves further attention. The analytical treatments of the linear Weibel instability used in Sect. 3 have been based on the linearized nonrelativistic Vlasov equation for the particle's phase space density, where the nonrelativistic particle momentum $p=m v$ is simply the product of the constant particle mass $m$ and particle velocity $v$, 
which neglects the additional Lorentz factor dependence $p=\gamma m v$ where

$$
\gamma=\sqrt{1+\frac{p^{2}}{m^{2} c^{2}}}
$$

Omitting the Lorentz factor dependence corresponds to the formal limit of an infinitely high speed of light $c \rightarrow \infty$ in Eq. (36). Obviously, at least for nonrelativistic plasma temperatures this omission has no profound effects on the growth rates. If we would, however, investigate the role of the Weibel instability at very early cosmological epochs, a relativistically correct dispersion theory is needed. The covariantly correct dispersion theory for unmagnetized anisotropic Maxwellian distributions of arbitrary composition has recently been developed [39]. The anisotropic bi-Maxwellian equilibrium plasma distribution functions are characterized by the two parameters $\mu_{a}=m_{a} c^{2} / k_{B} T_{\perp}$ and $\psi_{a}$ which in the nonrelativistic limit $\mu_{a}>>1$ correspond to the perpendicular thermal velocity $v_{t h, a, \perp}=c \sqrt{2 / \mu_{a}}$ and the temperature anisotropy $\psi_{a}=\frac{1}{2}\left[\frac{T_{a, \perp}}{T_{a, \|}}-1\right]$, respectively. We derived the dispersion relations of longitudinal and transverse oscillations in an unmagnetized anisotropic bi-Maxwellian plasma of arbitrary composition. They hold for any values of the plasma temperatures and the temperature anisotropy, and are the starting dispersion relations for investigating both nonrelativistic and relativistic temperature plasmas.

In the limit of nonrelativistic $\left(\mu_{a}>>1\right)$ anisotropic plasmas we expressed the transverse dispersion relation in terms of the plasma dispersion function 40]

$$
Z(f)=\frac{1}{\pi^{1 / 2}} \int_{-\infty}^{\infty} d x \frac{e^{-x^{2}}}{x-f}
$$

as

$$
0=\Lambda_{T} \simeq 1-\frac{c^{2} k^{2}+\sum_{a} \omega_{p, a}^{2}}{\omega^{2}}-\frac{1}{2} \sum_{a} \frac{\omega_{p, a}^{2}}{\omega^{2}}\left[1+\frac{2 \psi_{a}}{1-z^{2}}\right] Z^{\prime}(f)
$$

where $z=\omega / k c, Z^{\prime}(f)=d Z / d f$ and

$$
f=\frac{\omega}{v_{t h, a, \|} k} \frac{1}{\sqrt{1-\frac{\omega^{2}}{k^{2} c^{2}}}},
$$

In the formal limit of an infinitely high speed of light $c \rightarrow \infty$ this dispersion relation reduces to the standard noncovariant nonrelativistic form

$$
0=\Lambda_{\mathrm{T}, \infty} \simeq 1-\frac{c^{2} k^{2}+\sum_{a} \omega_{p, a}^{2}}{\omega^{2}}-\frac{1}{2} \sum_{a} \frac{\omega_{p, a}^{2}}{\omega^{2}}\left[1+2 \psi_{a}\right] Z^{\prime}\left(f_{\infty}\right)
$$

where

$$
f_{\infty}=\frac{\omega}{v_{t h, a, \|} k}
$$




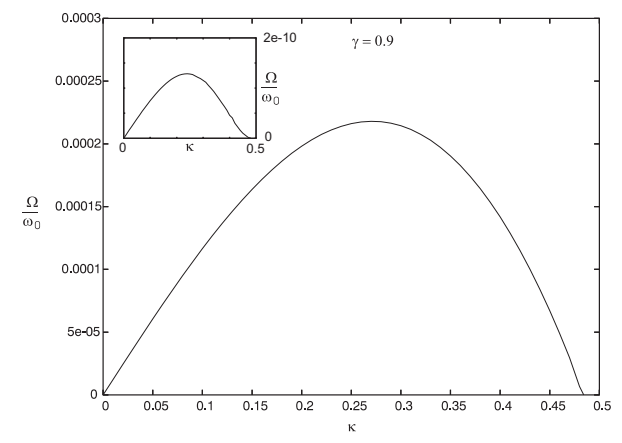

Figure 5. Growth rate of Weibel instability. From 34 .

\subsection{Aperiodic solutions}

Schaefer-Rolffs \& Schlickeiser 34 have discussed purely growing $(\omega=\imath \Omega)$ solutions of the transverse dispersion relation in case of the same anisotropy factor for all species. With $\omega_{0}^{2}=\sum_{a} \omega_{p, a}^{2}, \gamma=v_{t h, \|} / v_{t h, \perp}$ the covariant dispersion relation (38) reads

$$
G\left(\frac{\Omega}{v_{t h, \|} k} \frac{1}{\sqrt{1+\frac{\Omega^{2}}{k^{2} c^{2}}}}\right)=\frac{k^{2} c^{2}+\Omega^{2}}{\frac{k^{2} c^{2}}{\gamma^{2}}+\Omega^{2}}\left[1+\frac{k^{2} c^{2}}{\omega_{0}^{2}}+\frac{\Omega^{2}}{\omega_{0}^{2}}\right]
$$

with

$$
G(y)=1-\pi^{1 / 2} y e^{y^{2}} \operatorname{erfc}(y), \quad 0 \leq G(y) \leq 1, \quad \forall y \geq 0
$$

The limit $c \rightarrow \infty$ of Eq. (42) yields the noncovariant dispersion relation

$$
G\left(\frac{\Omega}{v_{t h, \|} k}\right)=\gamma^{2}\left[1+\frac{k^{2} c^{2}}{\omega_{0}^{2}}+\frac{\Omega^{2}}{\omega_{0}^{2}}\right]
$$

in the form discussed first by Kalman et al. 35. As we show now, these two dispersion relations provide basically identical solutions $\Omega(k)$ explaining why the covariant modifications play no significant role for nonrelativistic plasma temperatures.

With $\alpha=v_{t h, \|} / c$, dimensionless wavenumber $\kappa^{2}=\frac{k^{2} c^{2}}{\omega_{0}^{2}}$ and

$$
x=\frac{\Omega^{2}}{k^{2} c^{2}}=\frac{\Omega^{2}}{\omega_{0}^{2} \kappa^{2}} \in[0, \infty]
$$

the noncovariant dispersion relation (44) reads

$$
G\left(\frac{\sqrt{x}}{\alpha}\right)=R(x)=\gamma^{2}\left[1+\kappa^{2}(1+x)\right]
$$

while the covariant dispersion relation (42) becomes

$$
G\left(\frac{1}{\alpha} \sqrt{\frac{x}{1+x}}\right)=S(x)=\frac{\gamma^{2}\left[1+\kappa^{2}(1+x)\right](1+x)}{\left.1+\gamma^{2} x\right]}=R(x) \frac{1+x}{1+\gamma^{2} x}(47)
$$

The allowed range of anisotropies is $\alpha<<\gamma<1$ (i.e. $c>>v_{t h, \perp}>v_{t h, \|}$ ) so that the functions $S \geq R \geq \alpha$ for all wave numbers are larger than $\alpha$ implying that aperiodic solutions are only possible in the range $x \leq 1$. However, in this relevant 
solution range $x \leq 1$ the covariant function $S(x)$ and the noncovariant function $R(x)$ approach the same limit

$$
S(x \leq 1) \simeq R(x \leq 1) \simeq \gamma^{2}\left(1+\kappa^{2}\right)=\frac{1+\kappa^{2}}{1+\kappa_{\max }^{2}},
$$

where $\kappa_{\max }^{2}=\left(1-\gamma^{2}\right) / \gamma^{2}$. This explains that there is practically no difference between the covariant and noncovariant solutions for nonrelativistic plasma temperatures. The solution is shown in Fig. 5.

\section{Summary and conclusions}

We have demonstrated by analytical linear instability calculations and PIC simulations that counterstreaming electron-ion plasmas induced by cosmological structure formation magnetize the early universe by Weibel-type instabilities. The threshold condition for these instabilities requires intense streaming velocities $u>v_{t h}$ corresponding to large Mach numbers $M>43$ of the fully-ionized baryonic flows. There is good agreement between PIC simulations $\left(m_{p} / m_{e}=64\right)$ and analytical noncovariant instability estimates. The saturated magnetic fields reach 5 percent of the initial flow energy and can explain the existence of localized intergalactic magnetic fields of microGauss strength. For nonrelativistic thermal velocities there exists practically no difference between covariant and noncovariant plasma dispersion theory which can be verified by the now available covariant dispersion theory of unmagnetized anisotropic plasmas.

\subsection{Acknowledgments}

Partial support by the Deutsche Forschungsgemeinschaft through SFB 591 is gratefully acknowledged. I thank my coworkers J.-I. Sakai, U. Schaefer-Rolffs and P. S. Shukla.

\section{References}

[1] Y. Sofue, M. Fujimoto and R. Wielebinski, Ann. Rev. Astr. Ap. 24, 459 (1996).

[2] P. P. Kronberg, Rep. Prog. Phys. 57, 325 (1994).

[3] P. P. Kronberg, Phys. Today 55, 40 (2002).

[4] L. M. Widrow, Rev. Mod. Phys. 74, 775 (2002).

[5] M. S. Turner and L. M. Widrow, Phys. Rev. D 37, 2743 (1988).

[6] C. Caprini and R. Durrer, Phys. Rev. D 65, 023517 (2001).

[7] J. Eilek, astro-ph/9906485 (1999).

[8] E. Witten, Nucl. Phys. B 249, 557 (1985),

[9] C. Hill, D. N. Schramm and T. P. Walker, Phys. Rev. Lett. 59, 2493 (1987).

[10] J. P. Ostriker, C. Thompson and E. Witten, Phys. Lett. B 180, 231 (1986).

[11] D. Grasso and H. R. Rubinstein, Phys. Rep. 348, 163 (2001).

[12] E.N. Parker, ApJ 163, 255 (1971)

[13] E.N. Parker, Cosmical Magnetic Fields (Clarendon, Oxford, 1979).

[14] Ya. B. Zeldovich, A. A. Ruzmaikin and D. D. Sokoloff, Magnetic Fields in Astrophysics (Gordon and Breach, New York, 1983).

[15] M. J. Rees, QJRAS 28, 187 (1987).

[16] A. Brandenburg, A. and K. Subramanian, astro-ph/0405052 (2004).

[17] A. Gruzinov, ApJ 563, L15 (2001).

[18] N. Okabe and M. Hattori, ApJ 599, 964 (2003).

[19] R. Schlickeiser and P. S. Shukla, ApJ 599, L57 (2003).

[20] V. Springel and L. Hernquist, MNRAS 339, 289 (2000).

[21] R. Cen and J. P. Ostriker, ApJ 514, 1 (1999).

[22] E. Weibel, Phys. Rev. Lett. 2, 83 (1959). 
[23] B. D. Fried, Phys. Fluids 2, 337 (1959).

[24] J. Sakai, R. Schlickeiser and P. S. Shukla, Phys. Lett. A 330, 384 (2004).

[25] A. I. Akhiezer, I. A. Akhiezer, R. V. Polovin, A. G. Sitenko and K. N. Stepanov, Plasma Electrodynamics (Pergamon, Oxford, 1975).

[26] M. Medvedev and A. Loeb, ApJ 526, 697 (1999).

[27] F. Miniati, MNRAS 337199 (2002).

[28] R. J.-M. Grognard, Australian J. Phys. 28, 731 (1975).

[29] F. Califano, F. Pegoraro, S. V. Bulanov and A. Mangeney, Phys. Rev. E 57, 7048 (1998).

[30] Y. Kazimura, J.-I. Sakai, T. Neubert and S. V. Bulanov, ApJ 498, L 183 (1998)

[31] T.-H. Yang, J. Arons and A. B. Langdon, Phys. Plasmas 1, 3059 (1994).

[32] J. M. Wallace and E. M. Epperlein, Phys. Fluids B 3, 1579 (1991).

[33] D. Rye, H. Kang and P. L. Biermann, Astr. Ap. 335, 19 (1998).

[34] U. Schaefer-Rolffs and R. Schlickeiser, Phys. Plasmas 12, in press (2004).

[35] G. Kalman, C. Montes and D. Quemeda, Phys. Fluids 11, 1797 (1968).

[36] F. Miniati, D. Rye, H. Kang, T. W. Jones, R. Cen and J. P. Ostriker, ApJ 542, 608 (2000).

[37] D. Rye, H. Kang, E. Hallman and T. W. Jones, ApJ 593, 599 (2003).

[38] O. Buneman, in Computer Space Plasma Physics, Simulation Techniques and Softwares, edited by H. Matsumoto and Y. Omura (Terra Scientific, Tokyo, 1993), p. 67.

[39] R. Schlickeiser, Phys. Plasmas 11, 5532 (2004).

[40] B. D. Fried and S. D. Conte, The Plasma Dispersion Function (Academic Press, New York, 1961). 